\title{
La enseñanza sexista de los deportes rugby y fútbol
}

\author{
Sexist teaching of rugby and soccer \\ Juan Casajuis \\ Instituto de Investigaciones en Humanidades y Ciencias \\ Sociales (UNLP - CONICET). Facultad de Humanidades \\ y Ciencias de la Educación. Universidad Nacional de La \\ Plata, Argentina \\ jcasajus@fahce.unlp.edu.ar \\ (DD https://orcid.org/0000-0002-0839-5989 \\ Lorena Berdula \\ Instituto de Investigaciones en Humanidades y Ciencias \\ Sociales (UNLP - CONICET). Facultad de Humanidades \\ y Ciencias de la Educación. Universidad Nacional de La \\ Plata, Argentina \\ lberdula@fahce.unlp.edu.ar \\ (D) https://orcid.org/0000-0002-1678-1396
}

\section{ReSUMEN:}

Se propone indagar de forma preliminar las prácticas de la enseñanza en el rugby y fútbol examinando las categorías que emergen desde ellas y no a partir de lo que otros dicen que deberían ser, empleando nuestra propia voz al intentar explicarlas y describiendo lo que ocurre tal y como sucede. Este artículo pretende mostrar algunas líneas de propuestas didácticas para el aprendizaje del rugby y fútbol jugando de forma mixta- no binaria ${ }^{1}$ hasta una determinada edad ( 14 años en el rugby, 12 años en el fútbol), teniendo como consideración básica, la inclusión de sujetos diversos y de distintas condición física, bajo un contexto de actividades de enseñanza y aprendizaje basadas en las características que definen dichos deportes. Abordamos parcialmente concepciones de género que atraviesan el rugby y el fútbol, centrándonos en la voz de entrenadores y entrenadoras en ambos deportes. Es importante pensar la perspectiva de géneros y sexualidades, la interseccionalidad, los feminismos y transfeminismos para luego abordar la inclusión, los derechos humanos, la equidad, la igualdad de oportunidades y derechos en la enseñanza y acceso a la práctica del rugby y del fútbol, entre otros.

Palabras clave: Cuerpos, Prácticas, Rugby, Fútbol, Deportes no binarios.

\section{Abstract:}

This written advance proposes to investigate in a preliminary way the practices of teaching in rugby and soccer by examining the categories that emerge from them, using our own voice when trying to explain themand describing as it happens. This article aims to show some lines of didactic proposals for teaching rugby and soccer playing in amixed-non-binary way up to a certain age (up to 14 years old in rugby\& up to 12 years old in soccer), taking as a basic consideration, the inclusion of subjects of different sexes and physical condition, in acontext of teaching and learning activities based on the characteristics that define these sports. We partially address gender conceptions that cross rugby and soccer, focusing on the voices of coaches in both sports. It is important to think about the perspective of genders and sexualities, intersectionality, feminisms and transfeminisms to then address inclusion, human rights, equity, equal opportunities and rights in the teaching and access to the practice of rugby and soccer, among others. KeYwORDs: Bodies, Practices, Rugby, Soccer, Genres, Mixed sports, Non-binary sports. 


\section{INTRODUCCIÓN}

Se propone un análisis focalizado sobre las prácticas y algunos saberes pensando los efectos en la configuración y re-configuración de los cuerpos cis ${ }^{2}$ femeninos y masculinos (el abordaje de las identidades trans amerita otro escrito dada la histórica binarización de los reglamentos y el abordaje en el sistema escolar que ha excluido a las identidades diversas).

Las prácticas en el rugby y en fútbol están pobladas de un sinnúmero de conflictos y situaciones que hacen de los entrenamientos y partidos ámbitos sumamente complejos, muy difícil de describir teniendo en cuenta todas sus dimensiones. Se abordan parcialmente concepciones de género que atraviesan el rugby y el fútbol, centrándonos solamente en algunos aspectos particulares de las mismas.

Los problemas de géneros por binarización de la práctica se reflejan cada vez más en diversos ámbitos como el familiar y el laboral (Galofré, 2017). Pero no parece opinarse del mismo modo e intensidad en el ámbito educativo y menos aún, desde los deportes, por lo menos en países de habla hispana. Es por eso que proponemos ahondar en teorías que no han surgido desde esta área disciplinar, sino que provienen de las ciencias sociales y la filosofía.

Como así también, este artículo pretende mostrar algunas líneas de propuestas didácticas para el aprendizaje del rugby y el fútbol jugando de forma mixta- no binaria hasta una determinada edad (14 años en el rugby, 12 años en el fútbol), teniendo como consideración básica, la inclusión de sujetos diversas identidades y distintas condición física, bajo un contexto de actividades de enseñanza y aprendizaje basadas en las características que definen dichos deportes. El deporte es una herramienta educativa, se cree según Parise, Pagani, Cremascoli, \& Iafrate (2015) que transmite altos valores educativos y fomenta las capacidades individuales siendo también instrumento para la formación de los sujetos.

El deporte como disciplina ha sido al tamente enfocado desde sus inicios a destacar tanto la competitividad, el reto físico y la rivalidad como medio de superación. Sin embargo, más allá de pensarlo como una contradicción en el ámbito educativo (y formativo), ambos modos y sus objetivos conviven a tal punto que se puede separar del rendimiento como único fin, porque también es importante enseñar el beneficio del deporte resignificado como recurso orientando la posibilidad en promover habilidades de índole cooperativa y empática al ser deportes en equipo considerados socialmente como capital cultural (Bourdieu, 1997).

\section{Metodología}

\section{Género en singular y en plural.}

Al observar las prácticas en la enseñanza de los deportes como el rugby y fútbol observamos el ofrecimiento desigual para los varones en relación a las mujeres. Claramente las mujeres se han invisibilizado en esta práctica y las que han ofrecido resistencia al privilegio de los varones cis a la práctica del rugby y del fútbol, mientras que las mujeres no solo han pagado un costo muy alto como tener que deconstruir en sus cabezas el "no podes por ser mujer" y de poder hacerlo convivir con la resonancia de que "lo haces tan bien como un hombre ${ }^{3}$ ".Como también, no siendo suficiente, se las ha estigmatizado y condenado con conceptos como machonas, poco femeninas, tortilleras, locas, histéricas, desviadas, raras, solo como excusa para subestimar su participación en deportes con fundamentos patriarcales, androcéntricos y hegemónicos.

Pensamos concretamente en la organización y en la formación desde la niñez, las oportunidades dentro de los ámbitos formales como escuelas, colegios y Centro de Educación Física (CEF) como en ámbitos no formales sean los clubes, los municipios, los barrios, instituciones privadas, etc. que han discriminado y excluido la participación de niñas, jóvenes, mujeres como a los varones no hegemónicos ${ }^{4}$ al conformar organizativamente la enseñanza y las competencias. Creando los eventos competitivos y recreativos según 
sexo biológico en deportes esperables para uno u otro sexo. Siendo prácticamente imposible para las mujeres practicar rugby y fútbol en estas condiciones.

Si bien en el rugby y en el fútbol podemos encontrar ejemplos de clubes y ligas, son los menos los que permiten una práctica mixta, por ejemplo hasta los 12 años en fútbol como sucede en la liga infantil de Berisso y hasta los 14 años en la organización de la enseñanza en rugby ${ }^{5}$. Cuando esto sucede es porque los mismos padres o profesores se encuentran con la necesidad de que su hija o estudiante participe de la competencia. Otras razones son la empatía, por vínculos, por necesidad cuantitativa de completar los equipos o insistencia intensa de sus hijas.

Esto se explica en la construcción de la enseñanza de los deportes bajo el paradigma biologicista sexogenérico que ha binarizado a los deportes en femeninos y masculinos por una concepción reduccionista del rendimiento de los cuerpos sexuados binariamente, donde se explica que el mejor rendimiento lo tienen los cuerpos masculinos por su rendimiento hormonal y todo lo contrario los cuerpos femeninos que en una relación de poder no deberían acceder a esas posibilidades.

Paradójicamente se protegió a las mujeres por ser las que tienen la capacidad de fecundar olvidándose la situación particular que pasan durante los nueve meses, el momento del pujo o no del parto, su amamantamiento, en fin la crianza en general (podemos pensar en la jugadora de EE.UU. Alex Morgan que ha entrenado hasta los 4 meses de embarazo pero eso no se visibiliza). Con este criterio biologicista también se excluye pensar la existencia de mujeres que eligen no maternar porque al patriarcado y al mandato social no se le ocurre pensar en la capacidad de una mujer en elegir si ser madre o no siendo parte de los mandatos sociales. Incluso el ejemplo de los varones, mujeres trans e intersexuales podría ser ampliado en futuros textos

El concepto de género en singular al cual lo podemos definir como una construcción cultural y social binaria que se relaciona con el sexo designado al nacer que se lo relaciona con los modos y roles de ser varón hegemónicamente masculino y mujer hegemónicamente femenina, está todo dicho y hecho en el sentido que racionalmente encaja en dos baldosas como dice la escritora Gabriela Mansilla (2016, 2018), madre de una niñez trans, para la comprensión desde la razón parece una absoluta verdad pero para la auto percepción es una verdad falsa, ya que habrá tantas femineidades y masculinidades como sujetos somos.

Este binarismo no solo es funcional a la dominación masculina en términos de Bourdieu (2000) y a la perspectiva de género que abonaron los movimientos feministas, sino a la comodidad de la enseñanza tradicional y en particular a la enseñanza de la Educación Física reproductora de desigualdad y de binarismos.

Es de suma importancia correr el velo de lo binario y poner en tensión el binomio sexo-género para pensar y dar lugar a la posibilidad de la diversidad sexual en todas sus dimensiones (género, identidad de género, expresión de género, sexo biológico, orientación sexual).

Afirmar el término géneros en plural nos invita a incomodarnos y deconstruir filosóficamente nuestras prácticas, repensarlas, resignificarlas, reconfigurarlas, siendo un costoso trabajo personal y académico. ¿Qué se hace con toda la bibliografía escrita en masculino y en masculino universal producida hasta el momento si pensamos en desbinarizar la motricidad de los cuerpos para dar lugar en nuestras clases a otras corporalidades y corporeidades? Quedan invitadas/os a seguir haciendo el ejercicio de deconstrucción con otros elementos de la enseñanza.

Retomar como recurso ineludible la perspectiva de géneros y sexualidades será el modo de desandar el camino didáctico de la enseñanza de los deportes mixtos-no binarios en tanto deconstrucción filosófica, como el ejercicio de repreguntar en sentido inverso a los sujetos protagonistas, interpretemos este ejemplo ¿se nos ocurriría pensar que el jugador de rugby no tiene fuerza para jugarlo? En consecuencia ¿por qué lo preguntamos en las rugbiers? Pensando un ejemplo en fútbol, ¿̇se nos ocurriría pensar en la homosexualidad en el jugador como un problema para el equipo?, entonces ¿por qué la homosexualidad de las mujeres es un problema para el equipo? Claramente estos modos de pensar a las mujeres en el deporte manifiestan las relaciones de poder asimétricas del varón androcéntrico sobre las mujeres y las consecuencias que trae para 
la participación en las mismas. Para seguir teniendo poder se excluye con discursos supuestamente negativos hacia las mujeres.

La autora Judith Butler en sus libros Género en disputa (1990) y Deshacer el género (2006) entre otros, nos da cuenta cómo estas construcciones sociales y culturales del género que se enseñan y aprenden en las agencias o instituciones, disciplinan cuerpos binarios en tanto se espera un solo modo de ser varón y mujer performativos ${ }^{6}$, es decir el modo de actuar o caracterizar el género femenino para parecer y ser femenina o el género masculino para parecer y ser masculino.

Para confirmar las limitaciones del paradigma biologicista que instala el binarismo en el deporte podemos retomar la cita de Marta Lamas $(1997,1999)^{7}$ :

[...] en el sentido de afirmar que existe una paradoja epistemológica respecto al género. La paradoja es que el género es una verdad falsa pues, por un lado, la oposición binaria masculino/femenino es supraordenada, estructural, fundante y trasciende cualquier relación concreta; así masculino/femenino como reificadas de la diferencia sexual, son verdaderas. Pero, por otro lado, esta verdad es falsa en la medida en que las variaciones concretas de la vida humana rebasan cualquier marco binario de género y existen multitudes de casos que no se ajustan a la visión dual. (Virginia Goldner, 1991, pp 17)

Esto rompe con lo performativo cultural, con lo estereotipado. La paradoja epistemológica vine a explicar una verdad falsa y aparece la posibilidad manifiesta de la resistencia a los mandatos sociales y por ende las mujeres que quieren hacer rugby y fútbol en todas las instituciones.

\section{Saberes y protagonistas, sus representaciones}

Según expone Berdula (2015) los sentidos y significados que los profesores les otorgan al deporte y a su enseñanza como los principios y lógicas que los sostienen en esas instituciones y prácticas, se anclan sobre tres dimensiones centrales denominadas inicialmente "saber deportivo", "saber enseñar" y "saber institucional" 8 Es claro que al momento de desarrollar las prácticas entran en tensión los tres saberes de forma dialéctica, y el/ la entrenador/a reconfigura y resignifica sus propios saberes contextualizados (también el sistema deportivo) para situarlos en la enseñanza del deporte. Este hecho convierte en artesanal a la enseñanza, a la cual pensamos como la construcción de la propuesta educativa, su estilo, su impronta, en particular la de los deportes como el rugby y el fútbol.

$\mathrm{Al}$ pensar las tres dimensiones saber deportivo, saber enseñar y saber institucional, podemos distinguir cuales son las características y representaciones en las voces de sus actrices y actores, en este sentido interpretamos los discursos de las y los jugadores como la de las y los entrenadores/profesores.

En el saber deportivo nos encontramos con representaciones binarias de los saberes en el momento de desarrollarse, se observan en la organización en las diferentes categorías y niveles competitivos, sin atender la participación de las mujeres.

Este saber deportivo es resignificado por el saber institucional que es el que propone las condiciones situadas del deporte, en este caso rugby y fútbol con improntas propias fundadas en su historicidad y biografía institucional atravesada pos la idiosincrasia cultural barrial.

Mientras que los dos saberes anteriores son interpelados por el saber enseñar propiamente dicho que puede ser ejercido por ex jugadores y jugadoras (son las menos por la invisibilidad y falta de oportunidades que se le ha dado a las mismas), por idóneos, por entrenadoras/es y directoras/es técnicos con títulos habilitantes y las y los profesores en Educación Física que pueden sumar algunos de los roles anteriormente mencionados como la capacidad artesanal de enseñar.

Estos saberes nos muestran porque el porqué de los diferentes y disimiles objetivos y rendimientos al interior de ambos deportes. Lo que queda claro es que necesario enseñar de algún modo los deportes populares como el rugby y el fútbol legitimando la formación en diferentes niveles edades pero no lo hacen a ambos sexos. 
Destacamos que las instituciones están conformadas en su casi totalidad por varones cis hetero normados hijos del patriarcado (como todas y todos), explicando porque no se pueden pensar y pensar la práctica del deporte de otro modo. No se encuentra lo que no se va a buscar, como tampoco se acepta lo que nos va a incomodar.

Queda claro que el saber nos hace responsable y en este sentido quienes tenemos la posibilidad de dejar de reproducir desigualdades en los deportes somos las profesoras y profesores en Educación Física que podemos significar la importancia de la perspectiva de géneros y sexualidades en la enseñanza de los mismos.

Es hora que legitimemos desde el desarrollo de los contenidos desde los diseños curriculares la enseñanza de estos dos deportes en las escuelas, colegios y Centros de Educación Física (Gómez, 2002).

Para describir qué ha hecho la Educación Física con la enseñanza escolar de éstos deportes podemos situar la categoría de género en las prácticas como una categoría relacional, es decir según el Diccionario de Estudios de género y feminismos (Gamba \& Diz, 2007), "lo femenino y lo masculino se conforman a partir de una relación mutua, cultural e histórica" sin cuestionar lo que se reproduce de relaciones entre un sexo y otro donde se explica la reproducción sexo género como un par indisoluble que se refleja en las lógicas del rugby y del fútbol en tanto la organización de los mismos, como los entrenamientos, espacios edilicios, categorías, quienes participan, etc.

Llegamos a la conclusión que ningún juego o deporte es genérico o sexuado, se resignifica por medio de representaciones y configuraciones, según la biografía personal del entrenador/entrenadora que lo instrumenta como saber a enseñar, significado, o como contenido. Se puede decir que la intervención del entrenador/entrenadora condiciona las características sexistas o igualitarias de la utilización y trasmisión de los juegos y deportes como saber cultural y contenido (Berdula, 2015).

\section{Discusión}

Proponemos indagar las prácticas de la enseñanza en los dos deportes abordados desde las categorías que emergen de ellas y no a partir de lo que otros dicen que deberían ser, empleando nuestra propia voz al intentar explicarlas y describiendo lo que ocurre tal y como sucede.

Se describen cuáles son las diferentes concepciones de género, que utilizan las entrenadoras/entrenadores. En líneas generales las/los entrenadoras/es conciben y marcan una diferencia entre los géneros. Esto se evidencia en algunos dichos explícitos de algunos de ellas/os refiriéndose a que las mujeres "son de madera" y que no pueden hacer con los varones los entrenamientos mixtos, o como el entrenador " $\mathrm{B}$ " que se indigna porque tiene un grupo que según él no es capaz de comprender "ciertas cosas" (por su condición de mujer), también nombramos al entrenador " $\mathrm{C}$ " cuando dice que no se puede comparar a los varones y mujeres porque los varones son otra cosa (en referencia a que son superiores). Surge pues una necesidad de capacitar en perspectivas de géneros desde las instituciones del estado como el Curso virtual en Deporte y Género del Ministerio de Turismo y Deporte de la Nación en el mes de junio de año 2020.

Dentro de la institución madre del fútbol argentino AFA, el 19 de diciembre de 2019 se creó el área de Equidad y Género que entre otras funciones implementará capacitaciones y ya cuanta con el observatorio de géneros para observar entre otros indicadores, cantidad de jugadoras, Directoras Técnicas, entrenadoras, preparadoras físicas, árbitras, dirigentes, presidentas de clubes, entre otros roles, en todo el país para luego generar implementación de políticas en el deporte. Algunos números significativos del Observatorio del Área de equidad y género de AFA nos muestran que existen 3.200 jugadoras registradas AFA siendo 33.200 jugadoras en ligas federales.

En paralelo, la Asociación de Técnicos Argentinos (ATFA) realizó en septiembre de 2019 el Primer Congreso de Directoras Técnicas de Argentina, organizado por una comisión de mujeres y dictado también por mujeres de todo el país. Con una concurrencia de las más de 100 técnicas y técnicos que paradójicamente eran muy pocos. Cabe aclarar que el nombre del congreso ha logrado incomodar a los varones y muchos no 
han concurrido pensando que era solo para mujeres, cuando a la inversa se utiliza el masculino universal y las mujeres asisten. En el año 2020 se crea el Departamento de Equidad y Géneros y se realiza (vía zoom) el Segundo Congreso de Directoras Técnicas y la Especialización en Fútbol Femenino para las Escuelas de Técnicos de todo el país. Todo ello a pesar de la desigualdad entre los 18.000 técnicos varones en relación de las casi 100 mujeres.

También es necesario visibilizar la semi profesionalización del fútbol femenino argentino que cuenta con 8 jugadoras con contrato por equipo (con opción a más como lo hicieron clubes como San Lorenzo, Boca, etc.) siendo parte de sindicatos de jugadores y directores técnicos (ATFA, FAA). Las jugadoras cuentan con licencia por embarazo. Curiosamente la mayoría cuerpos técnicos son masculinos, incluidas todas las Selecciones Nacionales argentinas.

Debemos visibilizar como los clubes se han sumado a la creación del área o secretarías de Género y Diversidad en la mayoría de los clubes de primera división de fútbol y en cada vez más clubes de rugby. Reflejando en la actualidad los movimientos sociales que se vienen conformando políticamente desde hace tiempo con los feminismos, en contra de las violencias a las mujeres y diversidad sexual de Argentina. Acunados en los Encuentros Nacionales de Mujeres que llevan casi 35 años de realización marcando el inicio de las actuales manifestaciones de los movimientos de mujeres y diversidad.

Todos estos cambios manifiestos en los movimientos sociales han empoderado a las mujeres en la lucha de sus derechos y oportunidades, quedando como cita la Profesora Marta Antúnez ${ }^{9}$ los "deportes como último bastión de conquista feminista".

A pesar de ello podemos encontrar a otras/os entrenadores que intentan no demostrar esa forma de pensar a sus jugadores: los entrenadores " $\mathrm{D}$ " y " $\mathrm{E}$ " no marcan diferencias e intentan destacar lo más objetivamente posible lo positivo y lo negativo de los grupos que les tocan. A pesar, incluso, de los prejuicios previos como era el caso del entrenador " $F$ " con el grupo de rugby femenino.

El resto de los entrenadores no se ocupan demasiado en cuidarse en tal sentido, ya que permiten vocabulario soez y despectivo hacia el género femenino o hacen bromas con sus jugadores sobre el tema. Sin embargo, varios se muestran preocupados por el tema, hasta incluso el entrenador "C" propuso alternativas de integración muy interesantes, que nunca intentó llevar a la práctica.

En el ambiente del fútbol, a pesar de las críticas que se les escucha decir y exponer en charlas y capacitaciones con respecto al fútbol femenino, insisten en dirigir mujeres porque lo ven como otra conquista del privilegio patriarcal y como una salida laboral a futuro. Lo enriquecedor en cuanto a "lo social" o vincular de este escrito es la variedad de matices que son muy diferentes a las de un grupo solo de varones o mujeres. En cuanto a las capacidades condicionales no observan dificultades notables. Las mismas jugadoras y jugadores reconocen que no existe ningún tipo de diferencia de género, sino más bien, cuando se separa, opina o discrimina se hace en relación al nivel de juego de cada uno, las y los que juegan mejor por un lado, y las y los que juegan peor por el otro.

Si pensamos en el fútbol, encontramos que entre las y los futbolistas las diferencias son más en relación a la edad futbolística que a la edad madurativa y cronológica. Si esa niña, joven o mujer ha tenido en sus pies una pelota desde su nacimiento no tendrá ningún inconveniente en dominar el deporte en todas sus dimensiones; por lo contrario si los prejuicios de la familia pesaron más que el deseo de su hija de jugar al fútbol le costará adquirir las destrezas, habilidades y pericia que sus pares congéneres y varones. Mientras que por el contrario, el niño encontrará infinidades de lugares para realizar su aprendizaje de fútbol.

El fútbol mixto, como describimos más arriba, tendrá lugar según la idiosincrasia territorial. Las ligas de fútbol infantil masculino ven como un problema la incorporación de las niñas. Piensan que los entrenadores pueden tocar a las niñas, piensan que no cuentan con los vestuarios suficientes, en el momento de lesionarse, en la supuesta fragilidad e incoordinación de las niñas para jugar partidos y entrenarse, etc. Y si deciden dejarlas jugar con los niños, los separan por sexo salvo raras excepciones que podrán jugar mixto. Una frazada corta para los tiempos en que vivimos en diversidad. 
Si pensamos en la práctica del fútbol en las escuelas, se reproduce lo mismo que en la enseñanza en general en que conviven los modelos de las clases sexistas, mixtas y no binarias, es decir queda condicionada la enseñanza del fútbol a la propia biografía e historicidad de la profesora y profesor de turno para ese grupo. Cuando se abordan las clases sexistas, el argumento es que separa los varones de las mujeres porque los varones se aburren, reciben muchas patadas, no progresa, y las mujeres no son coordinadas, no quieren o no les gusta jugar a la pelota, lloran por nada, todos prejuicios sexistas. Mientras las clases mixtas juntan a niñas y niños pero en un momento determinado de la clase los separan, es como que, se tiende a realizar la entrada en calor, algún juego y el partido final los vuelven a separar. También se observan clases donde son igualitarias durante toda la clase y se reflejan las consignas, las actividades, la organización espacial siempre igualitarias.

$\mathrm{Al}$ respecto del rugby muchas experiencias de las ya nombradas se repiten. Muchas niñas y jóvenes no se acercan con la excusa de ser un deporte violento, prejuicios que acompañan desde ámbitos familiares. Por otra parte, los entrenadores (tradicionalmente quienes conducen las prácticas) manifiestan dificultades de poder llevar adelante las actividades. Este escrito se propone superador al entender que existen varias estrategias (Orlick, 2002; Zagalaz Sánchez, 2013) para iniciarse en el rugby, incluso los juegos como el Tag rugby ${ }^{10}$ es un medio de iniciación valedero. En esta modalidad deportiva, pueden practicar con reglas sencillas mujeres y varones juntos. La modificación de ciertas reglas del rugby, como puede ser el contacto, hacen del Tag rugby un deporte cercano y accesible a todos y todas. Este es otro medio que constituye un contenido relevante en los ámbitos nombrados en este escrito (clases de educación física, clubes, etc.) puesto que pone en juego valores tales como la coeducación, el espíritu de superación y la aceptación de unas reglas de juego.

Para finalizar este análisis, veremos las actitudes relacionadas al género que presentan los entrenadores al dar sus entrenamientos y partidos: existe una evidente desigualdad entre los 18.000 técnicos de fútbol varones en relación de las casi 100 mujeres con títulos oficiales.

Sin lugar a dudas, como se transcribe recuperando sus voces, es variado el comportamiento de cada entrenadora y entrenador. Conviven diferentes modos de entrenar (Casajús, 2020), con o sin perspectiva de géneros, con intensión pero sin lograrlo como están también quienes ni se lo preguntan. Algunos ni en los comentarios parecen ofrecer un trato diferente a los varones de las mujeres. Otros trabajan las mismas actividades con mujeres y varones, y en el momento de realizar algún tipo de discriminación para organizar un juego, lo hace en función de la habilidad y no del género. Se observa que quienes tienen formación mayormente pedagógica no incurren en estas conductas.

\section{Conclusiones}

Los deportes como el rugby y el fútbol están atravesados por una inmensa cantidad de conceptos, teorías y paradigmas. Por medio del presente trabajo se busca ahondar y acercar algunas aclaraciones de la relación existente entre estas disciplinas y las problemáticas de la categoría de género al momento de entrenar. Especialmente respecto a las concepciones que sujetos y entrenadoras / entrenadores manejan sobre estos temas.

La mayoría de las entrenadoras y entrenadores (por biografía e historicidad personal) consideran al rugby y al fútbol como disciplinas que tienen como objetivo principal el desarrollo de las capacidades condicionales(fuerza, resistencia y velocidad), y muy alejado de ello se encuentran otros objetivos de menor importancia, los relacionados con la integración, la recreación, la construcción de la ciudadanía, la educación por y para la libertad, el desarrollo de todas las cualidades corporales psicomotrices y sociomotrices, entre otras.

Proponemos escribir pautas de modo colaborativa para reflexionar críticamente sobre nuestras prácticas, como así incentivar nuevas experiencias también para colaborar en modificar la forma de pensar y relacionarse entre jugadoras y jugadores. La sobredimensión que toman las capacidades funcionales es una excusa que se utiliza en la sociedad y en los clubes para marcar las desigualdades entre los géneros. 
El abordaje en perspectiva de géneros pasa a un segundo plano dentro de los entrenamientos y partidos debido a la importancia desmedida que se le otorga al trabajo desde las capacidades condicionales y coordinativas. Pero que siempre aparece como el principal obstáculo, y en muchas oportunidades como un obstáculo insalvable bajo la mirada biologicista.

Muchos enfoques sociales que abordan la teoría de la interseccionalidad (Crenshaw, 1989), son subvalorados por las entrenadoras y entrenadores a no ser que sean muy evidentes, como por ejemplo, el caso de las violencias por ser mujeres o varones no hegemónicos como el bullying, o entre géneros. Los demás problemas sociales como los de géneros, religión, clase social, etc. son considerados solo en función de las facilidades o dificultades que presentan para trabajarlos desde las capacidades condicionales. Es decir, que mientras puedan trabajarse fácilmente dichos contenidos en los entrenamientos y partidos, las cuestiones sociales parecerían carecer de importancia.

Esto puede deberse a que las entrenadoras y entrenadores de ambos deportes no tienen suficiente capacitación en perspectiva de géneros, sexualidades y feminismos, importantes herramientas para abordar dichas problemáticas como tampoco la formación y diálogos actualizados con las teóricas sociales, fisiológicas, entre otras, que les permita redimensionar las cuestiones sociales.

Sin lugar a dudas, este escrito solo comenta ciertas premisas preliminares acercando algunas opiniones a las inquietudes planteadas en los objetivos, que deberá ser complementada por otros aportes desde distintas perspectivas. Sugerimos algunas certezas, otras descripciones y se propone un aporte de forma de revisar las propias prácticas. Permitirnos revisar lo que sentimos, qué somos y cómo fuimos formados cultural y académicamente posibilitará un trato reflexivo y crítico en las intervenciones de las prácticas al momento de elegir las estrategias para enseñar los deportes.

Deberíamos reflexionar atentamente sobre la posibilidad de construir nuevos saberes en una nueva instancia cultural y luego con formato pedagógico para convertir el deporte en contenido. En la misma línea, promover una pedagogía de las diferencias y una didáctica crítica inclusiva como posible camino a seguir educando desde y para la diversidad.

En síntesis, sería importante pensar la perspectiva de géneros y sexualidades, la interseccionalidad, los feminismos y transfeminismos para pensar la inclusión, los derechos humanos, la equidad, la igualdad de oportunidades y derechos en la enseñanza y acceso a la práctica del rugby y del fútbol, entre otros.

\section{Agradecimientos}

Deseamos realizar un especial reconocimiento al Profesor Adolfo Bertsch y al Profesor Mario Mamonde quienes con su apoyo y confianza han realizado un aporte invaluable en nuestra formación y docencia.

\section{REFERENCIAS}

Berdula, L. I. (2015). Escuela, deporte y género. De singularidades y diversidades. En O. Ron, J. L. Fridman (coord.), Educación fisica, escuela y deporte: [Entre] dichos y hechos. La Plata: UNLP FAHCE.

Bourdieu, P. (1997). Capital cultural, escuela y espacio social. México: Editorial Siglo XXI.

Bourdieu, P. (2000). La dominación masculina. Barcelona: Editorial Anagrama.

Butler, J. (1990). El género en disputa. El feminismo y la subversión de la identidad. Buenos Aires: Paidós.

Butler, J. (2006). Deshacer el género. Barcelona: Paidós.

Casajús, J. (2020).¿Cómo aprenden los entrenadores a enseñar rugby?: El caso de los entrenadores de rugby de M14 en los clubes del Gran La Plata. Tesis de posgrado (Magíster en Deporte). Universidad Nacional de La Plata. Facultad de Humanidades y Ciencias de la Educación. 
Crenshaw, K. (1989). Demarginalizing the intersection of Race and Sex: A Black feminist Critique of Antidiscrimiation Doctrine, Feminist Theory and Antiracist Politics. Feminist Theory and Antiracist Politics, University of Chicago Legal Forum, 139-167.

Curso Género y Deporte. (2020). Lo que no podés dejar de saber sobre género, masculinidades y violencia. Taller Online. Ministerio de Turismo y Deporte, Argentina. Disponible en: https://view.genial.ly/5ea17b40efce850db1f109 5c?fbclid=IwAR1LCcuYYDrKoxAnNUO-JrslpkqasxySRxLj06LHvWGhUvfd0jjSkO3lJck

Formación en perspectiva de género. (2016). Material de capacitación sobre Masculinidades. Secretaria de Derechos Humanos de la Provincia de Buenos Aires.

Galofré, P. (18 de diciembre de 2017). Construir la masculinidad de forma consciente. TEDx Reus. Recuperado de ht tps://www.youtube.com/watch?v=RhUYfwVb6dg

Gamba, S.; Diz, T. (2007). Diccionario de Estudios de género y feminismos. Buenos Aires: Biblos.

Gómez, R.H. (2002). Género y didáctica de la Educación Física-Subjetividad y cuerpo propio en la infancia. Educación Física y Deportes. Revista Digital. 8(47), Abril de 2002, Buenos Aires. http://www.efdeportes.com [Consulta: Noviembre 2004]

Lamas, M. (1999). Género, diferencias de sexo y diferencia sexual. Debate feminista, 10 (20) 84 - 106, octubre 1999

Lamas, M. (1997). La perspectiva de género. En: Taller Interdisciplinario sobre Género y Derechos Humanos (1o.: 1997 oct. 9-11: México, D.F.). México, D.F., Comisión Nacional de Derechos Humanos; Programa Interdisciplinario de Estudios de la Mujer del Colegio de México, 1997.

Mansilla, G. (2016). Yo nena, yo princesa: Luana, la niña que eligió su propio nombre. 1a ed. 6a reimp. - Los Polvorines: Universidad Nacional de General Sarmiento.

Mansilla, G. (2018). Mariposas libres. Derecho a vivir una infancia trans. Los Polvorines: Universidad Nacional de General Sarmiento.

Orlick, T. (2002). Libres para cooperar, libres para crear: (nuevos juegos y deportes cooperativos). Editorial Paidotribo. Madrid: España.

Parise, M., Pagani, A., Cremascoli, V. y Iafrate, R. (2015). Rugby, Self-perception and prosocial behaviour: evidence from the italian. "Rugby Project for Schools". Revista Iberoamericana de Psicologia del Ejercicio y el Deporte. Las Palmas, España.10 (1), 57-61.

Ron, O., Fridman, J. (coord.) (2015). Educación Física, escuela y deporte: (Entre) dichos y hechos. La Plata: Universidad Nacional de La Plata. Facultad de Humanidades y Ciencias de la Educación. (Diálogos en Educación Física; 2). Disponible en: http://libros.fahce.unlp.edu.ar/index.php/libros/catalog/book/44

Zagalaz Sánchez. M, Lara Sánchez, A. (2013). El deporte como vehiculo de integración. Wanceulen, editorial deportiva. Sevilla: España.

\section{Notas}

1 Tomamos la categoría de deportes mixtos por ser una realidad metodológica tradicional de construir la competencia y la formación binaria de los y las deportistas. Pero nuestra vasta experiencia nos invita a pensar una categoría social como el deporte no binario, que dé cuenta de los derechos de todas las identidades al derecho de la accesibilidad a los deportes y sus competencias.

2 Se denomina CIS (del mismo lado, opuesto a trans -del otro lado-) a los sujetos que su auto percepción coincide con el sexo biológico designado al nacer. Lo que se impuso como cuerpo hegemónico, varones con penes y mujeres con vulva desde el dispositivo sexo-género.

3 La cursiva es nuestra ya que preferimos el concepto de varón o varones porque cuando se piensa en el concepto de hombre como masculino universal puede llegar a incluir a las mujeres de modo que las termina invisibilizando. Lo mismo sucede cuando se utiliza hombre como concepto filosófico.

4 Se define a la masculinidad o varón hegemónico como la forma de masculinidad dominante y culturalmente autorizada en un orden social determinado, en el deporte lo relacionamos con rudeza, virilidad, fuerza, potencia, insensibilidad, por caso. 
5 Según el reglamento de rugby infantil UAR (2020), se puede jugar mixto hasta la categoría denominada Menores de 14 años (M14); este aspecto es desconocido por muchos actores.

6 La performatividad en Judith Butler se comprende como el modo de actuar los roles sociales binarios de ser estereotipadamente varón o mujer. Plantea que manifestarse de un modo u otro tiene relación con el sexo que determina al género culturalmente impuesto en relaciones de poder y lo cuestiona.

7 Marta Lamas (1997) en su escrito “Género: algunas prescripciones conceptuales y teóricas" nos permite abordar y ampliar el tema sobre dicotomía sexual.

8 Categorías que surgen en el grupo de Investigación de la que la autora es parte junto con Ron, Osvaldo; Fridman, Jorge, coordinadores (2015). Educación Física, escuela y deporte: (Entre) dichos y hechos. La Plata: Universidad Nacional de La Plata. http://libros.fahce.unlp.edu.ar/index.php/libros/catalog/book/44.

9 Creadora del Consejo de la Mujer en la Secretaría de Deportes de la Nación.

10 En las actividades del Tag Rugby, los jugadores y jugadoras llevan un cinturón de "tag rugby" y dos cintas pegadas con velcro a ambos lados. Consta de un equipo de 12 jugadores/as con 7 en el campo de juego. El equipo en ataque tiene que intentar marcar un try, se marcan puntos al apoyar la pelota detrás de la línea de try y antes de la línea de pelota muerta. Tendrá 5 oportunidades para marcar con cada posesión. Cada vez que la defensa quita una cinta, pierde una oportunidad. Cuando el equipo defensor (sin la pelota) quita la cinta a un jugador/a del equipo atacante (con la pelota), esto se considera un tackle. Cualquier forma de contacto no intencionada puede ser sancionada con un penal y/o cambio de posesión. 